Meta

Journal des traducteurs

Translators' Journal

\title{
Approche interdisciplinaire de la médiation linguistique à la frontière australe de l'empire colonial espagnol : deux outils méthodologiques
}

\section{Gertrudis Payàs Puigarnau et José Manuel Zavala Cepeda}

Volume 58, numéro 2, août 2013

URI : https://id.erudit.org/iderudit/1024174ar

DOI : https://doi.org/10.7202/1024174ar

Aller au sommaire du numéro

Éditeur(s)

Les Presses de l’Université de Montréal

ISSN

0026-0452 (imprimé)

1492-1421 (numérique)

Découvrir la revue

Citer cet article

Payàs Puigarnau, G. \& Zavala Cepeda, J. M. (2013). Approche interdisciplinaire de la médiation linguistique à la frontière australe de l'empire colonial espagnol : deux outils méthodologiques. Meta, 58(2), 261-278.

https://doi.org/10.7202/1024174ar

\section{Résumé de l'article}

La recherche historique sur l'interprétation pose le problème de l'absence de sources directes pour reconstruire les situations dans lesquelles des interprètes sont intervenus. Cependant, suivre la trace des interprètes institutionnalisés dans les textes coloniaux hispano-américains permet de trouver des indices et des références utiles dans ces reconstructions. Grâce à un travail interdisciplinaire (anthropologie historique et traductologie) sur les chroniques et les archives relatives aux négociations de paix hispano-mapuche entourant la frontière araucane, dans le sud du Chili (particulièrement au cours des XVII ${ }^{\mathrm{e}}$ et XVIII ${ }^{\mathrm{e}}$ siècles), nous proposons, dans le présent article, deux outils méthodologiques pour aborder l'interprétation en tant que pratique historique. Cette expérience nous permet de conclure que l'on peut mieux connaître les pratiques de médiation linguistique en passant par le dialogue interdisciplinaire. Du côté des études en interprétation, cette approche révèle des profondeurs peu explorées de cette dernière, et du côté de l'anthropologie historique, la médiation linguistique acquiert des contours plus précis en tant qu'objet d'étude. 


\title{
Approche interdisciplinaire de la médiation linguistique à la frontière australe de l'empire colonial espagnol: deux outils méthodologiques
}

\author{
GERTRUDIS PAYÀS PUIGARNAU* \\ Universidad Católica de Temuco, Temuco, Chili \\ gpayas@uct.cl \\ JOSÉ MANUEL ZAVALA CEPEDA* \\ Universidad Católica de Temuco, Temuco, Chili \\ jmzavala@uct.cl
}

\begin{abstract}
RÉSUMÉ
La recherche historique sur l'interprétation pose le problème de l'absence de sources directes pour reconstruire les situations dans lesquelles des interprètes sont intervenus. Cependant, suivre la trace des interprètes institutionnalisés dans les textes coloniaux hispano-américains permet de trouver des indices et des références utiles dans ces reconstructions. Grâce à un travail interdisciplinaire (anthropologie historique et traductologie) sur les chroniques et les archives relatives aux négociations de paix hispanomapuche entourant la frontière araucane, dans le sud du Chili (particulièrement au cours des $X \mathrm{XII}^{\mathrm{e}}$ et $\mathrm{XVIII^{e }}$ siècles), nous proposons, dans le présent article, deux outils méthodologiques pour aborder l'interprétation en tant que pratique historique. Cette expérience nous permet de conclure que l'on peut mieux connaître les pratiques de médiation linguistique en passant par le dialogue interdisciplinaire. Du côté des études en interprétation, cette approche révèle des profondeurs peu explorées de cette dernière, et du côté de l'anthropologie historique, la médiation linguistique acquiert des contours plus précis en tant qu'objet d'étude.
\end{abstract}

\begin{abstract}
Historical research on interpretation poses the problem of the absence of direct sources for the reconstruction of situations in which interpreters have intervened. However, in the process of tracking the presence of institutionalized interpreters in Hispanic-American colonial sources, we find indices and references in the documents that help us in these reconstructions. Through an interdisciplinary approach (historical anthropology and interpretation studies) to chronicles and archival material related to the Hispanomapuche treaties in the Araucanian frontier in Southern Chile (in particular during $17^{\text {th }}$. $18^{\text {th }}$ C.) we propose two methodological tools for approaching interpretation as a historical practice. This experience leads us to conclude that there is knowledge to be derived from past practice through interdisciplinary dialogue. From the point of view of interpretation studies, this approach takes us to depths of interpretation practice not often explored; from the perspective of historical anthropology, linguistic mediation is better clarified as an object of study.
\end{abstract}

\section{MOTS-CLÉS/KEYWORDS}

méthodologie, histoire de l'interprétation, mapuche, Chili, frontière araucane methodology, interpretation history, mapuche, Chili, Araucanian frontier 


\section{Introduction ${ }^{1}$}

Le présent article est la concrétisation de travaux de recherche entrepris dans le cadre de deux projets financés par le gouvernement chilien. Nous montrons comment le dialogue entre la traductologie et l'anthropologie historique peut combler des lacunes de ces deux disciplines relativement à l'étude de la médiation linguistique (conçue, bien entendu, en tant que médiation linguistico-culturelle) $)^{2}$.

Nous allons d'abord aborder certaines des difficultés que pose l'histoire de la médiation linguistique orale (c'est-à-dire l'interprétation des langues, ou, plus simplement, l'interprétation). Puis, nous recenserons quelques travaux publiés dans les domaines de l'histoire et de l'interprétation en soulignant les aspects pertinents pour les études des faits d'interprétation du passé, tout en relevant leurs limites.

Finalement, nous proposerons une liste des aspects relatifs aux faits d'interprétation, sous forme de protocole ou de fiche de traitement, centrés sur les personnages des interprètes, ainsi qu'un modèle pour aborder ces faits, en particulier ceux de la diplomatie hispano-mapuche: il s'agit d'accords de paix et d'amitié conclus régulièrement dans la frontière la plus australe des domaines coloniaux espagnols, soit la frontière araucane ou Araucanía. Cette approche est centrée sur les textes ou les procès-verbaux d'assemblées, les parlamentos, qui sont des textes faisant état du résultat de négociations qui se tenaient oralement, dans les langues espagnole et mapuche.

\section{Antécédents méthodologiques en histoire de l'interprétation}

Les recherches entreprises depuis quelques années en histoire de la médiation linguistique orale confirment la thèse défendue par plusieurs auteurs pour lesquels l'interprétation doit être considérée comme une pratique socialement située (Cronin 2002; Inghillieri 2003). Pour en comprendre les fonctions, les enjeux et l'étendue, nous adoptons une approche socio-historique, tout en nous appuyant sur les études déjà publiées dans notre propre discipline ainsi que sur la pratique professionnelle telle que nous la connaissons.

Il convient de souligner, comme d'autres l'ont déjà fait (notamment Baigorri 2006), que l'interprétation des langues, qui repose sur l'oral, ne laisse pas de trace. Les voix, une fois éteintes, à moins d'avoir été enregistrées par des moyens mécaniques, deviennent inaccessibles. $\mathrm{Si}$, comme résultat du fait d'interprétation, nous disposons d'un texte (un procès-verbal, un rapport, le texte final d'une négociation, ou autre), celui-ci n'est pas le registre de toutes les voix et ne peut donc pas être traité comme le reflet direct et transparent de ce fait d'interprétation, mais bien plutôt comme une réélaboration, un produit. En histoire, il sera bien entendu considéré comme une source permettant d'éclaircir et d'aider à comprendre certains aspects du fait, tout en représentant pour l'analyse un élément de complexité supplémentaire dont il faut tenir compte. Pour en extraire les traces que le processus et la forme de la médiation peuvent y avoir laissées, il faut donc lire entre les lignes et interpréter les textes en question.

En histoire coloniale, et plus particulièrement dans le cas de l'empire espagnol en Amérique, les sources secondaires (chroniques, histoires, rapports) font occasionnellement mention des médiateurs linguistiques. C'est par ces sources que nous connaissons les cas les plus emblématiques (par exemple Doña Marina, l'interprète 
de Cortès), sur lesquels l'information historique est relativement abondante, aussi bien que ceux des centaines d'interprètes, parfois nommés, parfois anonymes, qui ont fait le pont entre les Espagnols et la population autochtone dans tous les domaines de la vie civile, militaire et religieuse. Dans ces derniers cas, en particulier, la connaissance que nous pouvons avoir de ces personnages est partielle: parfois, nous ne pouvons savoir que ce qu'on leur a fait dire ou ce qu'on a voulu qu'ils disent. Dans une chronique coloniale où il est simplement dit: «moyennant un interprète on leur a fait savoir...», comme c'est souvent le cas, nous ne pouvons pas savoir comment l'interprète a livré le discours de son mandataire. Nous savons ce que son mandataire voulait communiquer aux Indiens, et cela, d'après le chroniqueur. Il n'y a donc que de faibles traces du passage ou de la présence du médiateur dans ces chroniques où celui qui parle est l'auteur du texte, lequel représente généralement la voix dominante dans l'interaction narrée (qu'il ait assisté ou non à l'événement). Les interprètes apparaissent dans les textes comme la voix de leurs maîtres, ce qui s'ajoute au peu de traces de leurs activités et s'avère déjà symptomatique du rôle limité que l'historiographie réserve encore aujourd'hui aux faits de médiation linguistique.

Cela dit, et indépendamment de ce que nous venons d'exposer, il convient de distinguer deux genres de difficultés méthodologiques ou, pour ainsi dire, lacunes: parfois elles sont dues au fait que ce que nous voulons connaître est peut être enregistré quelque part, mais ces renseignements n'ayant pas fait l'objet d'un intérêt historiographique particulier, ils font partie de la masse d'informations non traitée (par exemple, les données biographiques d'un interprète quelconque). Dans d'autres circonstances, les difficultés viennent du fait que ce que nous voulons connaître n'a jamais été enregistré, parce qu'à l'époque où les événements se sont produits, ce genre d'information ne comptait pas, ou parce qu'il n'y avait pas moyen de l'enregistrer (un énoncé ou un discours original, par exemple). Les lacunes du premier genre peuvent finir par être comblées, mais si ce que nous cherchons n'a jamais été enregistré, certaines questions resteront sans réponse, du moins avec nos outils de recherche actuels.

Rappelons que même les médiateurs ayant exercé dans des contextes de grande visibilité ou importance, et sur lesquels reposait en grande partie le succès des entreprises coloniales, ne sont pas mentionnés dans les chroniques. Nous ne pouvons donc ni savoir comment ils prêtaient leurs services ni, bien entendu, retracer leurs biographies. Les documents qui font état de ce qu'ils pensaient, croyaient ou disaient en dehors des situations de médiation sont également rares. Leurs responsabilités peuvent avoir été lourdes, mais la fiction de la communication immédiate et sans obstacles visibles dans les registres laisse entrevoir un consensus doxologique sur l'intérêt d'effacer ces présences ou, du moins, de les ignorer. Les tout premiers contacts entre Espagnols et Américains semblent parfois avoir lieu dans une même langue, comme si les uns et les autres se comprenaient tout naturellement: ainsi, «vinieron otros tres días a disfrutar la conversación de los cristianos» « «ils vinrent encore trois jours converser avec les chrétiens»], dit le récit de Jerónimo de Vivar, soldat de Pedro de Valdivia, le conquérant du Chili (Vivar 1608/1966: 24³).

Qu'il s'agisse de sources muettes ou de sources qui sont l'écho de la condition subsidiaire du médiateur ou de son appartenance au camp des vainqueurs, elles ne permettent généralement pas de reconstruire la voix même de l'interprète, les réactions des interlocuteurs, les défaillances de la communication, les bruits, les aspects «intratextuels», les formes d'énonciation, les capacités de mémoire, les connaissances 
linguistiques et extralinguistiques, le degré de dépendance ou d'indépendance à l'égard du commanditaire, les écarts, la littéralité, les pauses, le débit, l'expression, c'est-à-dire tous les aspects et faits saillants qui constituent l'expérience de l'interprète professionnel et qui, dans le cas d'interprètes du passé témoins ou figures importantes d'événements marquants de l'histoire, aideraient à avoir une vision plus complète des situations.

S'il est vrai qu'avant l'apparition des enregistreurs de son, cette information ne pouvait être recueillie ni analysée, quand il s'agit de faits très lointains, qui n'ont été racontés qu'une fois ou deux à leur époque (c'est-à-dire dont nous ne possédons qu'une ou deux sources contemporaines), tout essai de reconstruction comporte des risques de spéculation.

Comment s'étonner, dès lors, de ce que l'historien ou l'anthropologue non familiarisé avec la pratique de l'interprétation professionnelle de langues hésite à donner à ces faits du passé, si faiblement enregistrés, un poids et un sens, ou même une place précise dans ses analyses? Ainsi, à la raison coloniale qui tenait pour acquis que les Espagnols s'étaient bel et bien fait comprendre, on doit ajouter une cécité, voire un déni, même par ignorance, de la pratique de la médiation dans l'historiographie.

C'est dans ce contexte de difficultés méthodologiques, sources parfois d'un manque de traitement historiographique de la médiation linguistique orale, que nous croyons utile de proposer une démarche pour dépouiller les textes afin de mieux connaître les faits d'interprétation de langues du passé.

Quant aux travaux anthropologiques qui peuvent aider à éclairer les médiations linguistiques, il faut se référer à ceux qui ont cherché à comprendre les processus historiques de l'Amérique Latine du point de vue de l'autre, du vaincu. Nous pensons ici aux travaux de Nathan Wachtel sur les Andes $(1971 ; 1974 ; 1990)$ et à ceux de Serge Gruzinski sur le Mexique ou sur le métissage (1988; 1999), des auteurs qui questionnent les méthodes historiques traditionnelles et mettent en évidence les phénomènes de relations interethniques et de reconfigurations culturelles au centre desquels se trouvent la communication interlinguistique, les transferts de sens et les interprétations de différentes réalités. Dans la même veine, signalons les écrits des chercheurs canadiens Turgeon, Delâge et Ouellet sur les «transferts culturels» (Turgeon, Delâge et Ouellet 1996). La critique traductologique de l'ethnologie faite par Kate Sturge (2010) représente quant à elle un travail-charnière intéressant, qui a le mérite d'expliquer les articulations possibles entre anthropologie et traduction, sur les plans conceptuel et appliqué.

Quant aux recherches traductologiques à proprement parler, il faut dire que dans le corpus, déjà considérable et toujours croissant, de travaux de recherche en interprétation, les recherches historiques sont encore peu nombreuses, ce qui semble indiquer que dans la formation universitaire la réflexion historique est encore minoritaire, comme elle l'est d'ailleurs encore en traduction ${ }^{4}$. Hormis certains travaux épars (Santoyo 2003; Cáceres 2004) la filière la plus solide pour ce qui est du contexte hispanique serait celle entamée par Jesús Baigorri et Icíar Alonso à Salamanca, avec le groupe de recherche Alfaqueque de l'Université de Salamanca, qui cherche à mettre en évidence la longue tradition juridique espagnole concernant la diversité de langues et la pratique de la médiation linguistique et qui propose des articulations entre les modalités de la médiation linguistique dans différents contextes et diverses périodes historiques, ainsi qu'entre des modalités du passé et des modalités du présent ${ }^{5}$. 
Certains travaux non historiques, plus spécialement ceux qui se montrent ouverts à une perspective sociologique, représentent les éléments d'une charpente qui peut aider à soutenir l'édifice historique. Nous pensons notamment à ceux de Michael Cronin $(2002 ;$ 2006). Les recherches sur l'interprétation communautaire moderne et son contexte social et politique sont aussi utiles à nos besoins. Néanmoins, même s'ils sont d'ordre général, comme l'article de Bistra Alexieva (1997), qui propose une typologie des événements pour lesquels l'interprète sert de médiateur, les travaux théoriques ne sont pas immédiatement applicables aux situations du passé. Le sont encore moins les travaux d'ordre prescriptif ou ceux qui sont liés à des paires de langues précises, qui abondent dans la littérature scientifique du domaine. Par contre, certains travaux anciens, comme ceux de R. Bruce W. Anderson (1976), reproduits par Franz Pöchhaker et Miriam Shlesinger dans Interpreting Studies Reader (2001), et qui cherchent à appréhender globalement le phénomène de l'interprétation, peuvent être lus à la lumière des faits du passé.

Il est donc possible que la recherche historique sur la médiation orale ne puisse pas utiliser les cadres méthodologiques de la recherche actuelle axée sur le produit ou le processus, une recherche basée sur l'observation directe ou l'écoute d'enregistrements d'événements de médiation et orientée vers la recherche de la qualité professionnelle. Qui plus est, en toute logique, elle ne peut se passer des disciplines historiques, auxquelles, en revanche, elle peut apporter la perspective et la connaissance de la profession du point de vue de l'initié. Le dialogue entre traductologie et anthropologie historique que nous présentons ici nous semble donc pertinent dans la mesure où il permet non seulement de combler les lacunes en matière d'information factuelle propres à chacune de ces disciplines, mais parce qu'en agissant, pour ainsi dire, en tant qu'auxiliaires l'une de l'autre, les méthodes deviennent complémentaires et la connaissance de l'objet d'étude s'approfondit.

Ensemble, nous dessinons les contours de l'événement, un peu comme quand nous construisons un casse-tête en commençant par les bords de l'image et en allant vers le centre, où se trouvent les vides à combler. Cette reconstitution se fait à partir d'une analyse des sources historiques (les chroniques coloniales) ainsi que des documents qui sont le résultat direct d'une interaction à laquelle les interprètes ont pris part, par exemple les procès-verbaux des conférences tenues entre dignitaires de part et d'autre. À ce propos, nous nous sommes concentrés sur la figure de l'interprète institutionnalisé et sur la présence de l'interprète dans ces conférences, les parlamen$\underline{\text { tos }}^{6}$, au cours des $\mathrm{XVII}^{\mathrm{e}}$ et XVIII ${ }^{\mathrm{e}}$ siècles, dans la région de la frontière araucane. La formalisation de la médiation linguistique dans ce contexte singulier permet de mener des observations prolongées et de mettre à l'essai les outils d'analyse et de synthèse que nous proposons ici.

\section{L'interprétation institutionnalisée en contexte colonial}

La frontière constituée par la rivière Biobío, dans le sud du Chili, fut pendant trois siècles la frontière la plus australe de l'empire espagnol en Amérique ${ }^{7}$. Elle fut reconnue en 1641 par la paix de Quillín entre le gouverneur espagnol et l'ensemble des Mapuches de l'Araucanie, représentés par 162 caciques accompagnés de plus de 2000 guerriers $^{8}$. Cette paix, ratifiée quelques années plus tard (1647) lors d'un parla$\underline{\text { mento }}$ tenu au même endroit avec la même solennité, marque une limite importante 
de la conquête territoriale espagnole et la reconnaissance de fait du peuple mapuche (ou araucan) en tant que peuple indépendant et non soumis aux Espagnols ${ }^{9}$. La constitution de cette frontière fut le résultat du repli espagnol qui suivit le grand soulèvement mapuche de 1598-1602, par lequel se termina une première période de conquête espagnole au sud du Biobío (1552-1602). Pendant ce demi-siècle, les conquérants avaient mis à l'essai diverses stratégies pour assujettir les populations autochtones, les obliger au travail des encomiendas ${ }^{10}$ et les convertir. À l'échec de cette première conquête succèdera une période où la Couronne essaiera d'implanter la stratégie employée avec quelque succès dans la zone de la frontière chichimèque, qui correspond aux territoires situés au nord du Mexique actuel ${ }^{11}$. Il s'agit de la "guerre défensive", une stratégie consistant grosso modo à ne pas attaquer sauf si l'on est attaqué, tout en reconnaissant une limite au domaine espagnol (la raya, ou ligne), limite au-delà de laquelle les Indiens pouvaient vivre libres en tant que vassaux du roi non soumis au régime des encomiendas. Elle impliquait aussi le recours à des moyens pacifiques, notamment le dialogue, des concessions et des offres matérielles, pour convaincre les populations rebelles d'accepter la présence espagnole et de cesser les hostilités.

Le père jésuite espagnol Luis de Valdivia (1560-1642) sera le promoteur, porteparole et exécuteur principal de la "guerre défensive». Cet homme, traducteur ${ }^{12}$, interprète et mandataire d'interprètes, aura peut-être été le premier à saisir l'importance des échanges symboliques entre Espagnols et Mapuches. Muni de lettres de créance du roi, qui lui octroyaient des pouvoirs extraordinaires, tout particulièrement pour le recrutement d'interprètes ${ }^{13}$, et faisant fi des risques, il pénétrera dans le territoire ennemi pour établir des négociations directes avec ses chefs. Malgré ses efforts, cette politique ne lui survécut pas: elle touchait en effet aux intérêts politiques et économiques locaux, et portait préjudice aux profits dérivés de la guerre, notamment au trafic d'esclaves. Le XVII ${ }^{\mathrm{e}}$ siècle apparaît donc comme un mélange de guerre défensive et offensive avec, en arrière-plan, une politique déclarée de non-occupation des territoires indiens. Au gré des conjonctures politiques et économiques du Chili espagnol, les offensives militaires (les campeadas) alternèrent avec les négociations diplomatiques dont le père Valdivia sut si bien comprendre le protocole indien.

Même si la période dite de "guerre défensive» aura été en fin de compte brève, le système des parlamentos que le père Valdivia sut mettre au centre de la politique indienne se maintiendra pendant un siècle et demi et deviendra le fer de lance des rapports hispano-mapuches. Dès lors, les rapports officiels entre Espagnols et Indiens s'établissent dans un cadre proche du droit des gens avec la tenue périodique de grandes assemblées (une vingtaine de parlamentos dits generales entre 1641 et 1803, et des dizaines de parlamentos de moindre envergure), la nomination des interprètes et leur assermentation faisant partie des rituels d'ouverture. En effet, les efforts et gestes diplomatiques propres à la stratégie de négociation impliqueront le recours formel et incontournable aux médiateurs linguistiques: interprètes à proprement parler, mais aussi fonctionnaires connaisseurs des deux langues. C'est ainsi que les corps de médiation institutionnalisée dépendants de l'armée vont se développer et deviendront un phénomène unique dans l'histoire coloniale hispano-américaine.

Dans nos travaux sur les interprètes au cours de la période coloniale hispanoaméricaine, nous avons rencontré des formes diverses d'interprètes; parmi les interprètes institutionnalisés, les nombreux cas de nahuatlatos des Audiencias au Mexique 
sont bien connus, faisant l'objet de réglementation dans la législation (Leyes de Indias), ainsi que les cas d'interprètes moins officiels, également nombreux, qui accompagnaient les campagnes et explorations. Nous avions jusqu'ici peu de connaissance de l'activité d'interprétation dans les contextes diplomatiques, une activité moins fréquente. Lorsque la conquête avance sans difficultés, la figure habituelle de l'interprète est celle d'un indigène recruté plus ou moins violemment, utilisé et puis rejeté à l'arrivée dans un territoire où une autre langue est parlée, ses connaissances linguistiques n'étant plus utiles. Mais, lorsque la conquête s'arrête, quand l'ennemi oppose des obstacles permanents à son avancement, quand les rapports de pouvoir sont moins inégaux, l'interprète ad hoc ne suffit plus. C'est alors que nous trouvons dans les armées elles-mêmes la place et le poste de l'interprète, salarié et déclaré.

C'est la raison pour laquelle nous pensons que les frontières, lieux de tension, de rencontre et de conversation par excellence, bien plus que lieux de séparation, créent la figure de l'interprète institutionnel (qui coexiste avec d'autres figures de médiateurs, plus ou moins informels), et que cette figure et la pratique institutionnalisée de l'interprétation représentent des clés pour comprendre non seulement le fonctionnement de la médiation, mais les rapports interethniques et interculturels eux-mêmes.

\section{Protocole d'observation et analyse}

\subsection{Base de données des faits de médiation et de leurs interprètes}

Nous avons moins de difficulté à cerner les figures des interprètes institutionnalisés que celles des interprètes ad hoc, recrutés plus ou moins à l'improviste. Même sommairement, la présence de l'interprète officiel est constatée par l'historiographie coloniale. Et c'est en relisant les chroniques pour relever leurs noms et leurs contextes d'action que nous pouvons arriver à recomposer les situations de médiation. Un travail préalable (Payàs 2010), où nous avons exposé les articulations possibles entre les travaux théoriques modernes et le vaste corpus de témoignages des contacts interlinguistiques des siècles coloniaux établi par Emma Martinell (Martinell, Cruz Piñol et Ribas Moliné 2000), nous a permis de proposer certains éléments que nous pouvons transposer et appliquer à la lecture des chroniques chiliennes. Ils constituent les composants d'un protocole d'observation des cas de médiation linguistique pour notre contexte de frontière:

- Données biographiques de l'interprète;

- Formation linguistique: éducation formelle (collège, séminaire, etc.), informelle (commerce, travail, voyage, etc.), bilinguisme par parenté;

- Degré d'institutionnalisation: non institutionnalisé (interprète ad hoc), plus ou moins institutionnalisé (poste fixe, salarié, occasionnel, autres responsabilités) ou interprète-autorité (doté d'un mandat particulier);

- Partialité de l'interprète: sens unilatéral ou bilatéral;

- Formes de reconnaissance: contrats, accréditations, rituels d'assermentation, lieu où il se tient dans l'espace de négociation;

- Aspects juridiques et hiérarchiques: rapports hiérarchiques qu'il entretient, réglementation à laquelle il est assujetti;

- Aspects personnels, conjoncturels: famille, rapports, réseaux, influences;

- Autres présences dans la situation de médiation: interprètes, superviseurs, témoins, secrétaires, autres agents;

- Climat: tension, armes présentes, risques réels ou perçus. 
Chacun de ces composants est représenté par une case sur les fiches contenues dans la base de données que nous compilons: nous relevons les cas pertinents dans les chroniques ou documents d'archive, transcrivons les extraits des textes en entête de la fiche, puis nous remplissons les cases avec les informations ainsi obtenues. Il est rare que, pour un individu ou pour un fait de médiation, nous trouvions dans une seule source toute l'information permettant de remplir l'ensemble des cases. S'agissant de l'interprète institutionnalisé, nous réunissons pour un même individu des bribes d'information à partir de différentes sources, ce qui permet d'en faire un portrait contextualisé. Dans son ensemble, le travail quantitatif de collecte et de structuration en base de données nous aide à mieux comprendre les caractéristiques, la signification et le rôle de la médiation, ainsi qu'à tracer des régularités et à mieux définir les personnalités et leurs rapports immédiats avec les mandataires et leur entourage.

C'est ainsi, par exemple, qu'à l'aide de l'information obtenue de cinq sources différentes, lettres, chroniques et déclarations assermentées, nous pouvons donner aujourd'hui un portrait assez clair des interprètes Juan Bautista Pinto, Francisco Fris et Luis de Góngora et de leurs rapports avec le père Valdivia, leur mandataire, et déterminer autant que faire se peut la façon dont l'exercice de l'interprétation s'articulait avec les responsabilités militaires à la fin du Xvi ${ }^{e}$ siècle (Payàs et Zavala 2012).

Les procès-verbaux des parlamentos, que nous trouvons dès la fin du Xvi siècle, sont aussi une source importante d'information ${ }^{14}$. C'est à partir de ces manuscrits que nous avons pu déterminer le nom des interprètes officiels, tels que Juan Antonio Martínez, qui a servi dans tous les parlamentos $(1771,1774,1784,1793)$ tenus pendant la période d'influence de celui qui deviendra gouverneur du Chili, Ambrosio O’Higgins, et que nous avons pu découvrir les caractéristiques principales des modes de médiation linguistique employés dans ce contexte diplomatique ${ }^{15}$.

\subsection{L'approche du texte du procès-verbal du parlamento}

Comme nous l'avons signalé, malgré l'échec initial de la stratégie promue par le père Luis de Valdivia ${ }^{16}$, dès le milieu du XVII ${ }^{\mathrm{e}}$ siècle, les parlamentos deviennent les principales instances de négociation et d'échange entre les autorités espagnoles (gouverneur et chefs militaires) et les dirigeants mapuches. Ils se tiennent en langue mapudungun, à l'aide d'interprètes, un secrétaire du côté espagnol rédigeant le procès-verbal dans sa langue. Ce texte n'est pas traduit par écrit en mapudungun, la culture mapuche étant une culture orale qui donne tout son poids et sa légitimité à la parole énoncée de vive voix. Puis, à partir du manuscrit en espagnol, des copies faites à la main sont envoyées aux différentes autorités espagnoles concernées. Ces textes font état des moments saillants des négociations et reproduisent parfois les expressions même des négociateurs ainsi que de longs fragments de leurs discours. Ils nous renvoient donc l'écho des dialogues et des discours produits en langue indigène, traduits par l'interprète et mis en texte par le secrétaire. Ils sont le seul fait concret, tangible, la seule trace laissée par l'événement ayant permis des transactions portant sur des griefs, plaintes, échanges de captifs, normalisation du commerce, libre passage, acceptation de la présence des missionnaires en territoire araucan, etc., dans les deux langues. 
Ce texte polyphonique, portant les marques de la langue indigène et d'une langue castillane déjà teintée d'indigénismes (non seulement locaux, mais également provenant des Antilles et de l'Amérique centrale), marquée elle aussi par la réénonciation produite par l'interprète et la mise en texte du secrétaire, exprime une multiplicité de désirs et d'intentions. Ses destinataires sont les autorités espagnoles, que l'on doit convaincre de l'intérêt et du bien fondé de ces accords étrangement formels avec des indigènes - aux yeux hispaniques - bien moins «civilisés » que ceux des empires Inca ou Mexica, sur des territoires qui ne rapporteraient jamais des quantités importantes d'or ni d'argent.

Pour les Mapuches, le texte en tant que tel sera d'abord seulement considéré comme la façon dont les autres s'engagent. Ils ne le signeront jamais, même pas avec la croix, et rarement ils demanderont à en garder une copie ${ }^{17}$. Ces procès-verbaux étant encadrés dans un paratexte qui a la forme d'un rapport, nous pouvons penser qu'ils sont rédigés une fois l'assemblée finie. En dehors des accords à proprement parler (les capitulaciones), qui sont rédigés en prose juridique, le récit des événements et des prises de parole se fait dans un style formel, les chefs indiens recevant un traitement respectueux ou neutre. Sincères ou feignant la sincérité, les autorités espagnoles s'adressent aux dirigeants mapuches sur un ton paternaliste, teinté de bienveillance religieuse. La parole des Mapuches, traduite en espagnol par des hommes qui s'expriment dans les deux langues et qui connaissent bien les deux cultures, parsème le texte de nouveaux mots et de nouvelles acceptions et tournures de phrase (on demande aux chefs de s'abstenir de courir la flèche, c'est-à-dire d'appeler à la rébellion, ou on parle des soulèvements de la terre, où terre traduit l'acception du peuple du mot mapu).

Comment donc faire la lecture de ce document de façon à rendre compte aussi bien de la zone grise des échanges en présence de l'interprète, zone sur laquelle nous

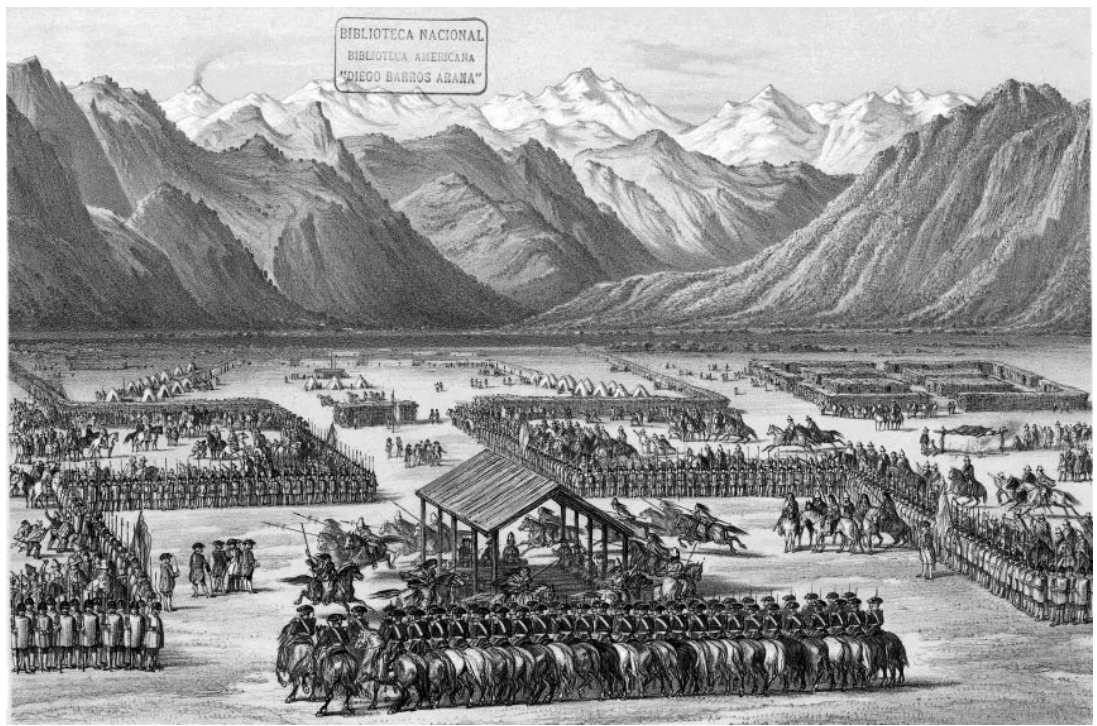


en savons si peu, que des contextes plus amples de la communication interethnique et de l'articulation entre l'événement et le contexte historique?

Partons d'une représentation d'un parlamento de l'époque: si l'on regarde de près la gravure publiée dans l'Atlas de Claude Gay (figure 1$)^{18}$, qui représente dans un ordre idéal, mais aussi dans tout son baroquisme, le parlamento de Negrete de $1793^{19}$, le site de production du document serait le centre de la ramada ou espace protocolaire, couvert de branches ( présence des autorités des deux camps, ainsi que de l'interprète et des ecclésiastiques surveillant celui-ci ${ }^{20}$.

Autour de cette ramada, sont étalés sur le vaste champ de Negrete, les effectifs militaires, de part et d'autre, ainsi que la chusma ou petit peuple indien, qui profitait de l'occasion pour fêter, échanger et concerter des alliances (plus de 2000 personnes dans le cas de Negrete, nourris et «choyés» [selon la pratique traditionnelle autochtone dont le nom fut traduit par agasajo par les Espagnols] par le trésor espagnol). Plus on s'éloigne de ce centre, plus on s'écarte de la négociation elle-même pour plonger le regard dans l'univers plus large de ces deux sociétés si différentes, qui acceptent de se rencontrer dans ces grandes cérémonies.

Sur cette image nous pourrions projeter un modèle à cercles, de caractère non pas synchronique, mais diachronique, composé de différents éléments (figure 2).

\section{FIGURE 2}

\section{Modèle en cercles concentriques pour l'approche du procès-verbal}

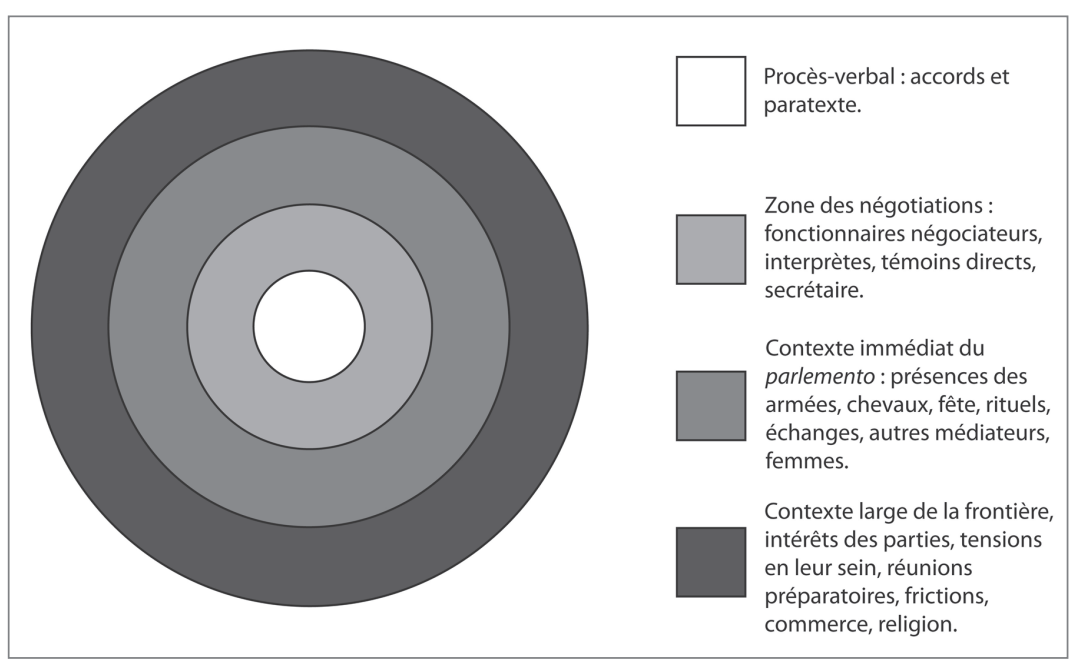

Le contexte élargi du front de conquête se trouverait dans le premier grand cercle: aspects politiques, juridiques, administratifs et économiques du projet colonisateur et des actions et réactions indiennes, dont fait partie la réglementation relative à la participation et à l'usage d'interprètes. Le deuxième cercle comprendrait les rapports plus précis entre l'armée espagnole et les groupes mapuches, les rapports frontaliers; c'est la zone où l'on doit rendre compte des frictions, des mouvements de population, du rôle de l'Église, du commerce, de la vie civile, du métissage, des mélanges et des transitions. Ici, figureraient aussi les accords préparatoires (appelés parlas) des grands parlamentos, réunions de moindre envergure qui se tenaient entre 
Espagnols et Mapuches pour déterminer l'endroit et l'ordre du jour du parlamento, les participants et la logistique. Feraient également partie de ce contexte les juntas de guerra ou rassemblements de guerre: des instances consultatives et résolutives réunissant l'état major et les autorités politiques et ecclésiastiques espagnoles qui devaient se prononcer sur le contenu des accords à signer au cours des parlamentos.

Dans le cercle suivant, nous nous trouvons directement dans le parlamento: pendant cette solennité étaient entendus les discours et propositions des autorités espagnoles et les réponses des dirigeants mapuches qui représentaient les quatre grandes alliances territoriales ou butanmapu ainsi que les unités politiques ou ayllarewe qui les composaient. La session d'ouverture commençait par un rituel indigène avec des éléments espagnols: les bâtons des caciques ${ }^{21}$ étaient mis ensemble, et une branche de canelo, l'arbre sacré des Mapuches, était portée en toute solennité et mise en évidence dans un endroit visible. Les médiateurs (interprètes et autres) prêtaient serment aussi lors de ces premiers rituels. Ensuite, le gouverneur espagnol, en tant que plus haute autorité du royaume, prenait la parole au nom du roi d'Espagne et, se faisant traduire par l'interprète, s'adressait aux représentants du camp indien pour leur annoncer les propositions (capitulaciones) qu'il souhaitait faire accepter.

À leur tour, les représentants indiens prenaient la parole en suivant un ordre préétabli selon leur hiérarchie. C'était le point de départ d'une série de discours, qui pouvaient se prolonger pendant deux jours ou plus, et dans lesquels ils réaffirmaient leur loyauté, formulaient leurs plaintes, menaçaient de sévir ou proposaient des réparations. Ces longs discours des caciques se tenaient dans un langage protocolaire et soutenu, très codifié, dont les arguments exprimaient toutes les raisons de faire la paix, d'éloigner la guerre et de ratifier l'engagement à l'égard des accords conclus. Ces paroles s'adressaient en premier lieu aux participants indiens et ensuite au gouverneur, de sorte que chacun des chefs indiens devait d'abord exprimer ouvertement sa position aux siens, la réponse au camp espagnol représentant la synthèse de toutes ces réponses. Ces prises de parole multiples étaient donc aussi une façon de construire un accord entre chefs indiens: entre ceux qui étaient déjà les alliés des Espagnols, et les autres, ceux qui, grâce au parlamento, allaient le devenir. Les procès-verbaux font état de ces longs discours de façon résumée, montrant souvent les difficultés, les tensions ou les moments de répit. La rhétorique indienne s'y trouve révélée, parfois traduite mot à mot, parfois en style indirect, tout comme la rhétorique espagnole, plus tributaire d'un discours paternaliste et religieux qu'à proprement parler juridique.

Après ces longs débats, les accords sont scellés par des rituels de compromis entre les appareils symboliques des deux cultures; au XVII ${ }^{\mathrm{e}}$ siècle, à tout le moins, il est fait mention explicite du partage et de la consommation, par les chefs de deux camps, des cœurs sanglants des chilihueque (lamas) sacrifiés, ainsi que de la mise à feu ou enterrement solennel des armes représentatives des Mapuches et des Espagnols. Les accolades, les tirs d'artillerie, les banquets et la distribution de cadeaux aux représentants indiens et à leurs femmes font également partie des formalités par lesquelles les parties font preuve de leur volonté d'engagement.

Quels étaient les rôles précis de l'interprète tout au long des journées du parla$\underline{\text { mento }}$ ? Les vides à reconstruire sont là :

- Quand commençait-il à parler, quand s'arrêtait-il, qui lui en donnait l'ordre?

- Quel degré d'autorité avait-il?

- Où se tenait-il, à quelle distance de ses confrères? 
- Servait-il de la même façon les Espagnols et les Indiens?

- Traduisait-il au pied de la lettre ou résumait-il, surtout pendant les longs discours des chefs indiens?

- Se tenait-il à égale distance des parties (facteur de latéralité)?

- Quelles pauses faisait-il?

- Était-il toujours seul?

- Lisait-il, écrivait-il?

- Quelles autres fonctions lui étaient confiées: messager, maître de cérémonies, consultant, agent, espion?

- A quel point dépendait-on de lui?

- Est-ce que les Indiens se servaient également de lui (secrètement ou ouvertement)?

- Qu'il serve les uns ou les autres, quelles étaient les compétences linguistiques de l'interprète?

- Quelle était sa connaissance de la culture et des formes protocolaires des parties?

- Est-ce que les discours des Mapuches étaient parlés, chantés, mimés, et comment étaient-ils rendus en espagnol, et vice-versa?

- Qui contrôlait l'interprète et comment? Quel âge avait-il ? Quel rang occupait-il dans l’armée?

- Était-il armé?

- Était-il payé? Toujours? Combien?

Hormis certaines données quant à la paie et au rang, les réponses à toutes ces questions ne se trouvent pas directement dans les chroniques ou les accords conclus. Il faut interroger ces documents pour en extraire ou déduire les informations qui nous permettent de combler ces vides et de dégager le portrait non seulement des individus médiateurs, mais de la médiation elle-même.

Dans notre modèle en cercles, le texte des accords se trouve au centre. Il a une double fonction: coucher sur papier les engagements ainsi narrer la façon dont les négociations ont été menées. Qui était là, qui disait quoi, quand et comment, à quelle heure les discours commençaient et s'arrêtaient chaque jour, et de quelle manière l'assemblée était levée. Ces quelque dix ou quinze pages présentant des caractéristiques à la fois d'un texte diplomatique et d'une chronique, voire d'une œuvre littéraire, contiennent non seulement des informations factuelles, mais également projettent sur l'ensemble de l'interaction interethnique des hypothèses et des certitudes relatives aux caractéristiques et à la nature même des rapports, ceux-ci étant «ficelés» tant bien que mal par l'entremise de ces interprètes et d'autres personnes bilingues qui jouaient le rôle d'intermédiaires.

Dans le cas du parlamento de Tapihue, tenu du 21 au 24 décembre 1774, qui appartient donc à la même période que celui de Negrete et dont le procès-verbal est particulièrement complet et détaillé, le contexte élargi comprend des événements importants tels que la récente expulsion des Jésuites des domaines espagnols (1767) et les rebellions de la période 1766-1770. L’arrivée du gouverneur Ambrosio O’Higgins, représentant de la monarchie des Bourbon, marque le début de nouveaux modes de relation avec la population indigène. Les Jésuites, qui avaient été les architectes des parlamentos, sont remplacés par les Franciscains dans leur rôle de témoins et de surveillants des négociations. Il y a donc du nouveau dans le contexte (la décennie 1770) de ce procès-verbal. Les nouveaux dirigeants mapuches sont les anciens rebelles de la décennie antérieure et ils entreprennent volontiers de longues journées de voyage vers la capitale pour s'entretenir avec les autorités espagnoles et s'assurer des avantages conclus. Ils en profitent pour rendre visite à leurs compatriotes nommés 
embajadores (en fait ces «ambassadeurs» jouent le rôle d'otages servant à garantir du côté mapuche le respect des accords), ainsi qu'aux enfants mapuches descendant de caciques étudiant dans les collèges de Santiago. Les réunions tenues à Santiago font aussi l'objet de rapports où les interprètes sont mentionnés, ainsi que parfois les hommes ayant servi à ce titre lors des parlamentos de la frontière. Ce contexte nous informe donc aussi bien des aspects historiques, c'est-à-dire, de l'histoire événementielle, que des aspects structuraux, c'est-à-dire des formes de rapports qui se sont consolidées durant cette période.

Quand nous considérons le deuxième cercle à partir du centre, qui représente les informations pouvant nous renseigner de façon plus précise sur la manière dont les interprètes ont agi et l'atmosphère des négociations dans lesquelles ils ont servi, le dépouillement du procès-verbal s'avère riche en détail: nous y trouvons précisés les rituels d'ouverture et la séquence des réunions. Nous y apprenons que, le premier jour, les interprètes (désignés par leurs titres officiels, le Lengua General et Capitán de Amigos) doivent prêter un serment de fidélité quant à leurs traductions des propositions des uns et des autres:

Sa seigneurie demanda qu'en sa présence et en présence de toute l'assemblée le Lengua General Juan Antonio Martínez et le Capitán de Amigos Don Blas Yañes prêtent serment, ce qu'ils firent au nom de Dieu notre seigneur et du signe de la Croix, selon le droit, et ils promirent de dire la vérité et de traduire fidèlement et littéralement tout ce que le Président proposerait aux Indiens et tout ce que ces derniers répondraient, et tout ce qu'ils avanceraient. (Parlamento de Tapihue 1774; traduction des auteurs ${ }^{22}$ )

Ensuite, les ecclésiastiques connaissant la langue sont exhortés à surveiller les interprètes :

Il exhorta immédiatement le Révérend Père Fr. Jubilé Fernando Villarreal de l'ordre Séraphique, qu'il fit pour cela venir du couvent de la Villa de San Joseph de Buena Vista, et les autres ecclésiastiques connaissant la langue des Indiens à signaler à Sa Seigneurie si le Lengua General leur proposait tout ce qui lui était ordonné de dire et s'il traduisait fidèlement leurs réponses. (Parlamento de Tapihue 1774; traduction des auteurs ${ }^{23}$ )

Les interprètes traduisent en langue mapudungun les articles qui sont soit lus, soit énoncés sans texte d'appui. Dans les deux cas, ils s'arrêtent à la fin de chaque article pour que les Mapuches aient le temps de s'assurer d'avoir compris:

Et tous les Indiens prirent connaissance des articles énoncés par le Lengua General et ledit Capitán de Amigos Don Blas Yáñez, de façon tout à fait individuelle, sans qu’on passe de l'un au suivant avant qu'ils ne manifestent être tout à fait instruits de ce qu'il leur était dit. (Parlamento de Tapihue 1774; traduction des auteurs ${ }^{24}$ )

Il est possible d'imaginer que la langue juridique dans laquelle ces articles sont rédigés du procès-verbal se transforme en paraphrases, ponctuées peut-être d'exemples illustratifs. Comme dans n'importe quelle négociation diplomatique faisant partie de toute stratégie politique, il est aussi possible d'imaginer que les Mapuches anticipent la teneur des demandes espagnoles, de la même façon que les Espagnols savent en grande partie ce que les Mapuches veulent obtenir de la négociation.

L'interprète servira aussi de maître de cérémonie, ouvrira les séances des deuxième et troisième jours et donnera la parole aux dignitaires mapuches venant des différentes divisions territoriales (reducciones): 
[Le deuxième jour] le Lengua General présenta le cacique Gobernador de la Misión de Santa Fe, Don Ygnacio Levigueque, qui avait été choisi par le cacique Gobernador de Colgue Don Christoval Chuequelemu, et les autres caciques de son Butalmapu, qui appartenait au Sargento Mayor, pour qu'il parlât au nom de tous. (Parlamento de Tapihue 1774; traduction des auteurs ${ }^{25}$ )

[Le troisième jour] le Lengua General présenta de nouveau le dit Gobernador Don Ygnacio Levigueque, qui donna la parole au cacique Gobernador de la reducción de Santa Juana, don Pedro Guaiquinir. (Parlamento de Tapihue 1774; traduction des auteurs $^{26}$ )

Des détails, apparemment insignifiants, rappellent des pratiques modernes, et le fait que le métier s'exerce, pour l'essentiel, de la même façon depuis toujours: en raison, selon le procès-verbal, de sa fatigue, Juan Antonio Martínez, qui est l'interprète titulaire officiel, est, au quatrième jour, remplacé par un autre interprète. Néanmoins, il est précisé que Martínez n’a pas quitté les lieux, mais est resté pour aider son remplaçant et veiller à ce que tout soit bien compris:

[L]e dit cacique Gobernador don Francisco Neculbud se leva et se rendit à l'endroit où se tenait le faisceau de Bâtons accompagné d'un capitanejo (guerrier mapuche) et du Capitán de Amigos Don Juan Rey, qui agissait comme Lengua General, les caciques lui ayant demandé de remplir ces fonctions et l'ayant désigné pour ce ministère le deuxième jour du Parlamento, après avoir constaté que le titulaire Don Juan Antonio Martínez était fatigué. Ce dernier resta néanmoins aux côtés du dit Don Juan Rey et du dit capitán Don Juan [ou Blas] Yáñez pour que les uns signalent ce que les autres pouvaient oublier et que Sa Seigneurie ne manque jamais la moindre intelligence de tout ce qui se disait. (Parlamento de Tapihue 1774; traduction des auteurs ${ }^{27}$ )

Grâce à ces quelques fragments du texte, même s'il s'agit d'un document dont les auteurs et les destinataires appartiennent à la partie espagnole, il nous est possible de reconstituer certaines scènes de l'histoire et de nous rapprocher des mentalités présentes ou observables de part et d'autre dans la ramada.

\section{Conclusion}

L'historiographie des conquêtes impériales présente une grande quantité de données relatives à l'interprétation; souvent il ne s'agit que de menus détails, des noms cités au passage, des mentions sans intérêt apparent. Nous savons pourtant que le besoin de communiquer rendait indispensable l'entremise de médiateurs de toutes sortes: du simple passeur, commerçant ou scribe au quotidien, à l'interprète assermenté ou chargé des affaires indigènes au plus haut niveau politique.

Les relations entre Espagnols et Mapuches dans le contexte particulier de la frontière de l'Araucanie coloniale permettent d'observer la pratique de l'interprétation institutionnalisée sur une longue durée, pratique qui se développe dans les domaines diplomatique et militaire et dont la signification sociale et politique est, de ce fait, notable.

Le protocole et le modèle à cercles que nous décrivons dans le présent article ont été élaborés pour faciliter les observations et organiser les informations, et sont le fruit d'un dialogue interdisciplinaire dont nous montrons ici l'intérêt et la pertinence.

Effectivement, nous croyons pouvoir conclure que le travail conjoint de l'anthropologie historique et de la traductologie permet de mieux connaître les circonstances 
et caractéristiques des situations où les interprètes sont intervenus. Les affinités entre les deux disciplines pour la compréhension des phénomènes d'intersection culturelle, l'intérêt de l'anthropologie historique pour le point de vue de l'autre et les processus d'interaction socioculturelle, ainsi que celui de la traductologie pour une meilleure description et une compréhension accrue des situations et des personnages qui constituent le territoire propre des études d'interprétation ne peuvent que porter fruit. Comparativement aux études consacrées à l'histoire de la traduction, centrées sur les textes et requérant des compétences linguistiques et traductologiques spécialisées, celles qui portent sur l'histoire de l'interprétation présentent moins d'entraves au dialogue interdisciplinaire. La nature de l'objet d'étude - individus ou situations historiques faisant partie des contextes larges de l'histoire politique et culturelle $-s^{\prime} y$ prête plus aisément.

C'est ainsi que les deux outils que nous présentons, c'est-à-dire, le protocole d'observation et le modèle à cercles, permettent des interprétations utiles pour les deux disciplines. La connaissance des contextes et la pratique de l'observation et de l'analyse des sources propres à l'histoire et à l'anthropologie offrent aux études d'interprétation la possibilité de dépasser le stade de la description anecdotique, partielle ou isolée, ou du portrait hagiographique, et de proposer aux théorisations modernes des assises sur le plan diachronique.

En même temps, l'histoire de l'interprétation, et donc le regard de la traductologie, peut enrichir l'anthropologie historique et l'histoire en leur transmettant les connaissances de l'initié, c'est-à-dire celles ayant trait au métier, aux conditions, aux compétences, aux origines et aux impacts des faits d'interprétation: des connaissances qui peuvent empêcher l'invisibilisation des faits de médiation linguistique tant par l'ignorance que par une idéalisation irréfléchie.

\section{NOTES}

* Departamento de Lenguas y Traducción, Núcleo de Estudios Interculturales e Interétnicos (NEII), Universidad Católica de Temuco; Grupo Alfaqueque, Universidad de Salamanca.

** Departamento de Antropología, Núcleo de Estudios Interculturales e Interétnicos (NEII), Universidad Católica de Temuco.

1. Le présent article est une version approfondie d'un travail présenté dans une communication de Gertrudis Payàs au XXIII ${ }^{\mathrm{e}}$ Congrès annuel de l'Association canadienne de Traductologie, Université Concordia, Montréal (28-30 mai 2010), qui portait sur la méthodologie de la recherche en traductologie. Il fait partie des recherches entreprises dans le cadre des projets de recherche Fondecyt Regular 1090459 (2009-2011) et 1120995 (2012-2014). En ce qui concerne José Manuel Zavala, ce travail s'inscrit aussi dans les projets Fondecyt Regular 1090405 (2009-2011) et 1120857 (2012-2014). Nous remercions, pour leurs suggestions, nos collègues Clara Foz, Fabien Le Bonniec et Iveth Carreño, qui ont lu et révisé le manuscrit.

2. Ce projet interdisciplinaire est constitué de trois axes: traductologie, anthropologie historique et philosophie. Dans le présent travail, nous nous concentrons sur le dialogue traductologieanthropologie historique.

3. Pour une discussion sur la fiction de la communication, voir Alonso (2003).

4. Le bulletin du Conference Interpreting Research Information Network (CIRIN), qui recense les publications et les thèses universitaires, permet de connaître les axes de recherche privilégiés dans les études d'interprétation.

5. Voir le volume d'essais publié en 2010 par le Grupo Alfaqueque (Grupo Alfaqueque 2010), qui présente un regard diachronique sur la médiation linguistique.

6. José Manuel Zavala (2000) consacre un chapitre (VI) à la définition et à la caractérisation de l'institution du parlamento. 
7. Elle subsista après l'indépendance du Chili, opposant alors le peuple mapuche à la nouvelle république. L'assimilation totale ne se fit qu'à la fin du XIX ${ }^{e}$ siècle, au coût d'un grand nombre de victimes. Actuellement, le peuple mapuche continue de défendre ses terres, à l'instar d'autres peuples autochtones des Amériques.

8. Voir Archivo General de Indias (AGI), Ch.4, Minuta de 1643; Diego de Rosales (1674/1877-1978), f. [1].

9. C'est ce traité que les Mapuches invoquent toujours dans leurs revendications contemporaines de reconnaissance politique et territoriale.

10. L'institution, typiquement coloniale, de la encomienda (du verbe encomendar: recommander), mettait un certain nombre d'Indiens sous la tutelle d'un Espagnol (encomendero) pour qui ils travaillaient et qui s'engageait à les protéger, les instruire et les évangéliser.

11. Gertrudis Payàs et Icíar Alonso (2009) proposent que les modes de médiation avec interprète utilisés dans la zone de la frontière hispano-arabe des $\mathrm{XIII}^{\mathrm{e}}-\mathrm{XV}^{\mathrm{e}}$ siècles soient considérés comme l'antécédent des les interactions avec interprète dans l'histoire coloniale espagnole.

12. Le père jésuite Luis de Valdivia, qui arrive au Chili en 1593 à l'âge de 32 ans, écrit et publie la première grammaire et le premier vocabulaire de la langue mapuche, ainsi que des langues millcayac et allentiac, aujourd'hui disparues, et les premières traductions de textes de doctrine.

13. Provisión del Marqués de Montes Claros du 29 mars 1612, reproduite dans Diego de Rosales (1674/1877-78): II, p. 532.

14. C'est en particulier l'objet des projets de recherche dirigés par José Manuel Zavala: réunir les sources documentaires des parlamentos et effectuer le travail archéologique nécessaire pour établir autant que possible les lieux où ces assemblées se sont tenues. Les premières conclusions de ce travail ont été publiées dans Zavala (2012).

15. Lordre protocolaire suivi dans les parlamentos a été décrit et caractérisé par Zavala (2005). D’autres aspects sont étudiés dans Payàs (2012) et Payàs et Garbarini (2012).

16. L'attitude du père Valdivia s'opposera à la raison pragmatique qui imposait la représentation de l'Indien comme un Autre, barbare, sauvage et irréductible. Valdivia semble avoir passé outre les instructions reçues des plus hautes instances. De plus, le fait qu'il ait peut-être été peu habile visà-vis des autorités locales et moins diplomatique encore vis-à-vis de l'armée, qui en fait représente l'autorité dans la zone de la frontière, transforma son action, conciliatoire, en obstacle aux intérêts de la guerre et à toute une économie du conflit, dont beaucoup tiraient profit. Valdivia, semble-t-il, avait déjà causé des ennuis à ses supérieurs lors de son mandat comme recteur du Colegio de Santiago (1595-1602), ce qui fut attribué à son humeur mélancolique («aunque hombre de mucha religión, estaba sujeto a grandes melancolías, por lo cual habían padecido muchas amarguras sus subditos del colegio "; cité dans Zapater 1992: 21). Il sera victime d'une campagne de discrédit et même de diffamation, auxquelles ses interprètes prendront part. Ce cas a fait l'objet d'une communication de G. Payàs lors du VII Congreso Chileno de Antropología en 2010 et d'un article (Payàs et Zavala 2012).

17. Ce désintérêt vis-à-vis du papier ira jusqu'à la méfiance : c'est à cause des «papiers» qu'ils perdront leurs terres au XIX et $\mathrm{XX}^{\mathrm{e}}$ siècles.

18. GAY, Claude (1854): Atlas de la historia física y política de Chile, Paris: Impr. E. Thunot, 35.

Disponible librement sur WikipediaCommons. Consulté le 15 mars 2013, <http://commons. wikimedia.org/wiki/File:Parlamento_Negrete(1793).jpg>.

Claude Gay (1800-1873) est un naturaliste français.

19. Le parlamento de Negrete de 1793 est l'un des plus importants de la période. Outre la documentation d'archive le concernant, il est aussi décrit par un voyageur de l'époque, George Vancouver, dans A Voyage of Discovery to the North Pacific Ocean and Round the World (1791-1795). Lauteur a en effet été l'hôte du gouverneur Ambrose O'Higgins, qui lui en a fait le récit. La traduction française date de 1802 et est de P. F. Henry.

20. Pour les modes de surveillance de l'interprète, voir Payàs et Garbarini (2012).

21. Les bâtons étaient octroyés aux seigneurs mapuches (caciques ou ülmen) par le gouverneur espagnol, représentant l'autorité royale, en reconnaissance de leur fidélité et de leur rang. Le gouverneur en portait également un, qui était déposé dans le même faisceau. De cette figure dérivera un dédoublement du nom indigène pour désigner le seigneur: huinca ülmen (lit. seigneur étranger, ou blanc) sera le seigneur coopté de cette façon par l'autorité espagnole, tandis que le mapu ülmen (lit. seigneur de la terre) sera le seigneur local traditionnel. Voir FebRés, Andres (1765/1882): Diccionario araucano-español; ó sea Calepino chileno-hispano por el p. Andrés Febrés, de la Compañia de Jesús. Buenos Aires: Impr. por J. A. Alsina, p. 89. 
22. «[m]andó su señoría que en su presencia, y en la de todo el concurso se les recibiere Juramento al Lengua General Don Juan Antonio Martínez y al Capitán de Amigos Don Blas Yañes, que lo hicieron por Dios nuestro señor, y una señal de la Cruz, según forma de Derecho, bajo del qual prometieron decir verdad, y traducir fiel y literalmente cuanto el Señor Presidente propusiere a los Indios, y éstos respondieren, como lo más que adelantasen.»

Parlamento de Tapinue (1774): Archivo General de Indias, Ch. 189, f. 501.

23. «[i]nmediatamente exhortó al Reverendo Padre Jubilado Fr. Fernando Villarreal del orden Seráfico, que trajo a este fin del convento de la Villa de San Joseph de Buena Vista, y demás personas eclesiásticas inteligentes en el idioma de los indios a efecto de que adviertiesen a Su señoría si el Lengua General les proponía todo lo que se le mandava decir, y si traducía fielmente sus respuestas. » Parlamento de Tapinue (1774): Archivo General de Indias, Ch. 189, f. 501.

24. «Y enterados todos los indios de los expresados artículos por medio del Lengua General y de dicho Capitán de Amigos Don Blas Yáñez, con toda individualidad sin que se pasare de uno a otro antes de que manifestaren estar perfectamente instruidos de lo que se les decía.»

Parlamento de Tapinue (1774): Archivo General de Indias, Ch. 189, f. 508v.

25. «[El segundo día] le presentó el lengua general al cacique Gobernador de la mision de Santa Fe Don Ygnacio Levigueque, que habían elegido el cacique Gobernador de Colgue Don Christoval Chuequelemu, y los demás caciques de su Butalmapu perteneciente al Sargento Mayor para que hablase a nombre de todos.»

Parlamento de Tapihue (1774): Archivo General de Indias, Ch. 189, f. 509v.

26. Texte original en espagnol: «[El tercer día] presentó nuevamente el Lengua general al mencionado Gobernador Don Ygnacio Levigueque, quien dio la voz al cacique Gobernador de la reducción de Santa Juana don Pedro Guaiquinir.»

Parlamento de Tapinue (1774): Archivo General de Indias, Ch. 189, f. 510v.

27. «[S]e levantó el enunciado cacique Gobernador don Francisco Neculbud, y pasando al sitio en que permanecia el hacecillo de Bastones acompañado de un capitanejo, y del Capitán de Amigos Don Juan Rey que hacía de Lengua general por haberlo pedido y elegido para ese Ministerio el Segundo dia del Parlamento los caciques a causa de haber conocido fatigado al propietario Don Juan Antonio Martínez, quien sin embargo se mantuvo inmediato al mencionado Don Juan Rey y al capitán Don Juan [ou Blas]Yáñez por que advirtiesen unos lo que se olvidase a los otros, y no careciese Su Señoría de la más leve inteligencia de quanto se decía.»

Parlamento de Tapinue (1774): Archivo General de Indias, Ch. 189, f. 510v.

\section{RÉFÉRENCES}

Alexieva, Bistra (1997): A typology of Interpreter-Mediated Events. The Translator. 3(2):153-174.

Alonso, Icíar (2003): Ficción y representación en el discurso colonial: El papel del intérprete en el "Nuevo Mundo". In: Ricardo Muñoz Martín, éd. Actas del I Congreso Internacional de la Asociación Ibérica de Estudios de Traducción e Interpretación (Granada, 12-14 février 2003). Vol. 1. Granada: AIETI, 407-419).

Anderson, Robert Bruce W. (2001): Perspectives on the Role of the Interpreter. In: Franz PöchHacker et Miriam Schlesinger: The Interpreting Studies Reader. London/New York: Routledge, 209-217.

BAIgorri, Jesús (2006): Perspectives on the History of Interpretation. Research Proposals. In: Georges Bastin et Paul Bandia, éd. Charting the Future of Translation History. Ottawa: Presses de l'Université d'Ottawa, 102-109.

CÁceres, Ingrid (2004): Breve historia de la Secretaría de Interpretación de Lenguas. Meta. 49(3):609-628.

Cronin, Michael (2002): The Empire Talks Back: Orality, Heteronomy and the Cultural Turn in Interpretation Studies. In: Maria Tyмосzко et Edwin Gentzler, éd. Translation and Power. Amherst/Boston: University of Massachusetts Press, 45-62.

Cronin, Michael (2006): Translation and Identity. Oxford/New York: Routledge.

Grupo Alfaqueque (2010): Los límites de Babel. Ensayos sobre la comunicación entre lenguas y culturas. Madrid/Frankfurt: Iberoamericana Vervuert.

GruZINSKI, Serge (1988): La colonisation de l'imaginaire. Sociétés indigènes et occidentalisation dans le Mexique espagnol. Siècles XVI ${ }^{e}-X V I I^{e}$. Paris: Gallimard. 
GruZinski, Serge (1999): La pensée métisse. Paris: Arthème Fayard.

Inghillieri Moira (2003): Habitus, Field and Discourse. Interpreting as a Socially Situated Activity. Target. 15(2):243-268.

Martinell Gifré, Emma, Cruz Piñol, Mar et Ribas Moliné, Rosa, éd. (2000): Corpus de testimonios de convivencia lingüística (ss. XII-XVIII). Kassel: Ed. Reichenberger.

PayÀs, Gertrudis et Alonso, Icíar (2009): La mediación linguistica institucionalizada en las fronteras hispano-mapuche e hispano-árabe: ¿un patrón similar? Historia. 42:185-201.

PAYÀs, Gertrudis (2010): Tras la huella del intérprete en la historia colonial hispanoamericana. In:Grupo Alfaqueque. Los límites de Babel. Ensayos sobre cultura y comunicación. Madrid/Frankfurt: Iberoamericana Vervuert.

PAYÀs, Gertrudis (2012): Acercamiento traductológico a la mediación lingüística en la Araucanía colonial. In: Gertrudis PaYÀs et José Manuel Zavala, éd. La mediación lingüístico-cultural en tiempos de guerra: cruce de miradas desde España y América. Temuco: Universidad Católica de Temuco/Universidad de Salamanca, 19-36.

PAYÀs, Gertrudis et Garbarini, Carmen Gloria (2012): Riesgos y control del intérprete: lecciones de una crónica colonial para la historia de la interpretación. Onomazein. 25:345-368.

Payàs, Gertrudis et Zavala, José Manuel (2012): Al filo del malentendido y la incomprensión. El Padre Luis de Valdivia y la mediación lingüística. Historia (Santiago). 45(1):69-90.

Pöchhaker, Franz et Schlesinger, Miriam, éd. (2001): The Interpreting Studies Reader. London/New York: Routledge.

Rosales, Diego de (1674/1877-1978) : Historia General del Reino de Chile. Valparaíso: Imprenta del Mercurio.

Santoyo, Julio César (2003): Un quehacer olvidado: Los intérpretes-traductores de navíos. Quaderns de Filología. Estudis Linguístics. 8:1-21.

STURGE, Kate (2010): Representing Others. Translation, Ethnography and the Museum. Manchester: St. Jerome Publishing.

Turgeon, Laurier, Delâge, Denis et Ouellet, Réal, éd. (1996): Transferts culturels et métissages Amérique/Europe, $\mathrm{XVI} I^{e}-\mathrm{X} \mathrm{X}^{e}$ siècle. Sainte-Foy/Paris: Presses de l'Université de Laval/ L'Harmattan.

VIVAR, Jerónimo de (1608/1966): Crónica copiosa y verdadera de los Reynos de Chile. Santiago: Fondo Histórico y Bibliográfico José Toribio Medina.

Zapater, Horacio (1992): La búsqueda de la paz en la Guerra de Arauco: Padre Luis de Valdivia. Santiago: Editorial Andrés Bello.

Zavala, José Manuel (2000): Les indiens mapuche du Chili. Dynamiques inter-éthniques et stratégies de résistance, XVIIIe siècle. Paris: L'Harmattan.

Zavala, José Manuel (2005): Aproximación antropológica a los parlamentos hispano-mapuches del siglo XVIII. Austerra. 1-2:49-59.

Zavala, José Manuel (2012): Los parlamentos hispano-mapuches como espacios de mediación. In: Gertrudis PayÀs et José Manuel Zavala, éd. La mediación lingüístico-cultural en tiempos de guerra: cruce de miradas desde España y América. Temuco: Universidad Católica de Temuco et Universidad de Salamanca, 151-162.

Wachtel, Nathan (1971): La vision des vaincus: les Indiens du Pérou devant la Conquête espagnole, 1530-1570. Paris: Gallimard.

Wachtel, Nathan (1974): Lacculturation. In: Jacques Le Goff et Pierre Nora, dir. Faire de l'histoire: 1. Nouveaux problèmes. Paris: Gallimard, 124-146.

WACHTEL, Nathan (1990): Le retour des ancêtres. Les Indiens Urus de Bolivie XX ${ }^{e}-\mathrm{XVI}{ }^{e}$ siècle. Essai d'histoire régressive. Paris: Gallimard. 